microquasars ${ }^{10}$ - whether catalogued or not - are responsible for the feature around 620 $\mathrm{GeV}$. And let's not forget that a completely new type of astrophysical object could also produce the detected electron excess; after all, pulsars were discovered only in 1967, and until 1992 we were blissfully unaware of microquasars.

However, all is not lost in our attempts to determine the real source of the ATIC electron feature. Luckily, the expected spectral signature of electrons from nearby KK WIMP annihilation is very distinctive: it shows a gradual rise to a sharp peak and a 'cliff-like' drop-off at the high-energy end (see Fig. 4 on page 364). With a longer observation, or a bigger detector, which would markedly increase the number of electrons detected, one ought to be able to say with some confidence whether the ATIC feature is more like a regular bump (for example, from an astrophysical source) or the sharp, discontinuous feature expected from nearby dark-matter KK WIMP annihilation. In fact, existing $\gamma$-ray telescopes, both on the ground and in space, should also be able to search for this possible discontinuity in the spectra of integrated diffuse emission they detect.

In the near future, neutrino telescopes on the scale of cubic kilometres, such as IceCube, buried deep beneath the cold, clear Antarctic ice, could also aid our sleuthing for dark matter ${ }^{11}$. And we may even have our very own dark-matter factory soon: the Large Hadron Collider is due to start regular operations in the next year or so, and its data will be combed through thoroughly in search of any dark-matter signals.

Squeezing the dark-matter problem from both sides is certainly the way to go: astrophysicists staring up and particle physicists working deep underground may finally unveil the secrets of the dark side. And if it turns out that the ATIC detection really is due to an elusive WIMP about 600 times the mass of a proton, it will go to show just how truly insignificant we are - the wimpy, overweight dark side may have us beaten.

Yousaf M. Butt is at the National Academy of Sciences, Washington DC, on leave from the Harvard-Smithsonian Center for Astrophysics, 60 Garden Street, Cambridge, Massachusetts 02138, USA.

e-mail:ybutt@cfa.harvard.edu

Chang, J et al. Nature 456, 362-365 (2008)

2. Massey, R. et al. Nature 445, 286-290 (2007)

3. Spergel, D. N. et al. Astrophys. J. Suppl. Ser. 170, 377-408 (2007).

4. Milgrom, M. Preprint at http://lanl.arxiv.org/ abs/0801.3133 (2008)

5. McGaugh, S. Science 317, 607-608 (2007)

6. Disney, M. J. et al. Nature 455, 1082-1084 (2008).

7. Cheng, H.-C. et al. Phys. Rev. Lett. 89, 211301 (2002).

8. Torii, S. et al. Preprint at http://lanl.arxiv.org/ abs/0809.0760 (2008)

9. Hooper, D., Blasi, P. \& Serpico, P. D. Preprint at http://lanl arxiv.org/abs/0810.1527 (2008)

10. Heinz, S. \& Sunyaev, R. Astron. Astrophys. 390, 751-766 (2002).

11. Hooper, D. \& Baltz, E. A. Annu. Rev. Nucl. Part. Sci. 58, 293-314 (2008).

\section{DNA SEQUENCING}

\section{Mammoth genomics}

\author{
Michael Hofreiter
}

\section{Reconstruction of most of the genome sequence of the woolly mammoth illustrates how such investigations will pave the way for a deeper understanding of the biology and evolution of extinct species.}

How times change. Within the space of less than a decade, the development of highthroughput technologies has transformed the task of sequencing a mammalian genome from the years-long, multimillion-dollar endeavour it was originally, to a project that can be performed by an individual laboratory within a few months. Such is the breathtaking extent of progress that it has now been possible to sequence the nuclear genome of the extinct woolly mammoth (Fig. 1) almost to completion.

This achievement is described by Miller et al. on page 387 of this issue ${ }^{1}$. Stretches of mammoth DNA from a cellular organelle, the mitochondrion, which has its own small genome, have been sequenced previously. But tackling the 4 billion to 5 billion base pairs in the much larger and biologically informative mammoth cell nucleus posed a different order of challenge.

Ancient DNA - DNA obtained from fossils up to about 100,000 years old - is highly fragmented, is present in only trace amounts, and is usually swamped by bacterial and fungal DNA. So the idea of sequencing the complete genome of an extinct species was long unthinkable, given that for 30 years the only large-scale method available, the Sanger sequencing method $^{2}$, was not adequate for such a task. However, in 2005 a new way of sequencing DNA was published ${ }^{3}$. Known as the 454 method, it initially had a throughput of 20 million base pairs per run - an increase of two to three orders of magnitude compared with Sanger sequencing. Since then, this method's throughput has increased to about 100 million base pairs and three other types of sequencing approaches have been launched - Solexa, SOLiD and HeliScope. These methods produce as much as 10 billion base pairs of sequence data in a single run. But all of them are 'shotgun' methods, which prohave quite high error rates. So, to obtain a reliable sequence for each nucleotide position, a genome needs to be sequenced to multiple coverage, which Miller and colleagues estimate would be around 10-20-fold for the mammoth. Given the rapid increase in sequencing throughput, it is possible that even this will soon be achieved.

These developments have both changed the way we think about molecular genetics and shown how we might finally get a handle on the genomes of extinct species. Not least, some of the drawbacks of the new technologies for studying modern DNA are advantages when it comes to ancient DNA. Thus, whereas the Sanger method allows individual stretches of up to 800 base pairs to be determined in a single reaction, the new techniques yield much shorter sequences, sometimes as few as 30 base pairs ${ }^{4}$. This is a disadvantage when dealing with modern DNA. But it does not matter with ancient DNA, which is mostly fragmented duce rather short sequencing reads; some also

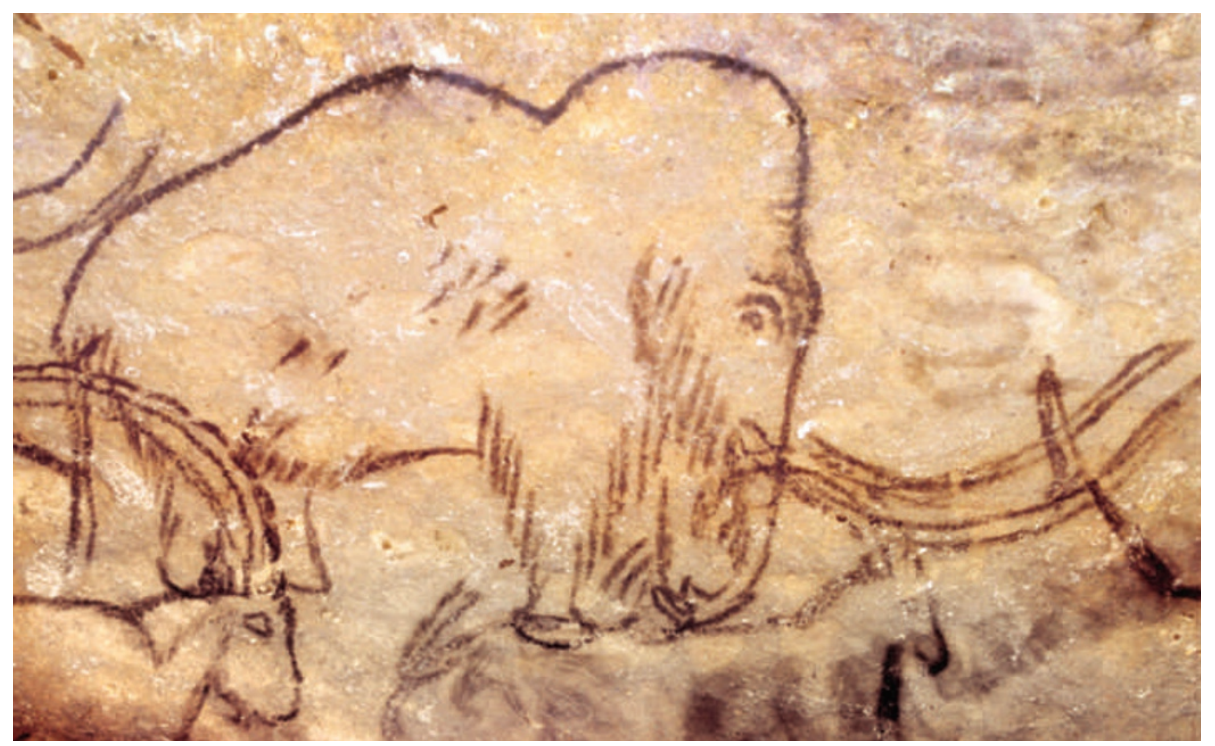

Figure $1 \mid$ Ancient blueprint. This wall painting of a mammoth and ibex is in Rouffignac Cave, Dordogne, France, and is dated to around 13,000 years ago. 
into pieces shorter than 100 base pairs.

Researchers were quick to exploit the new possibilities. Only months after the 454 technology became available, it was applied to mammoth genomics in a paper ${ }^{5}$ that reported 13 million base pairs of sequence - about 1,000 times more than were covered in the first ancient-genomics study with Sanger sequencing ${ }^{6}$. In that paper $^{5}$, published in January 2006, the authors also announced their plan to sequence the mammoth genome to completion. Miller and colleagues ${ }^{1}$ now describe about $70 \%$ of the mammoth genome, and so go a long way to achieving that goal.

Miller et al. were aided immensely in their task by the fact that, unusually for extinct organisms, some specimens of woolly mammoths have been frozen in permafrost. This is an ideal setting for preserving DNA, and, moreover, for preserving hair, which is an ideal source of DNA for sequencing ancient genomes. If hair still contains DNA, almost all of it will belong to the extinct species, and will not be of bacterial or fungal origin, as is often the case with bones. Thus, the authors needed to sequence a total of 'only' 4.1 billion base pairs to obtain about 3.3 billion base pairs of mammoth DNA. They calculate that the total mammoth genome, estimated at some 4.7 billion base pairs, would have been 1.4 times bigger than the human genome.

Although the mammoth genome is larger than the human genome, the DNA substitution rate seems to be smaller - this is the rate at which one nucleotide replaces another, and so is a measure of evolutionary change. The mammoth genome differs from that of its close relative the African elephant by as little as $0.6 \%$. This is about half the difference between human and chimpanzee, although the two elephant species diverged at about the same time as human and chimpanzee, and probably even slightly earlier (see Fig. 3 of the paper ${ }^{1}$ on page 389 ). For some reason, the substitution rate in the nuclear genome of elephants is much lower than in humans and great apes, a result mirrored in the mitochondrial genomes ${ }^{7}$, where humans and great apes also show a substitution rate more than twice as high as that in the elephant species. As nuclear and mitochondrial genomes are replicated by different enzymes, it remains unclear why both genomes evolve more slowly in elephants than in humans and great apes.

The draft mammoth genome sequence is too fragmented and error-prone to allow standard gene prediction. Nonetheless, Miller and colleagues identified several protein-coding positions that are unique to the mammoth compared with 50 other vertebrate species. The presence of such mammoth-specific differences is not surprising: it is to be expected that each mammalian species contains unique amino-acid substitutions compared with a limited number of other species. For example, the 52-amino-acid fragment of the ATP2C1 protein not only contains an amino-acid substitution unique to the mammoth, but a further two that are unique to the tenrec and the two-toed sloth, respectively. Similarly, the position in the 30-amino-acid fragment of the protein C1orf190, at which the mammoth differs from other placental mammals, also has amino-acid substitutions in the ground squirrel and kangaroo rat. Although Miller and colleagues argue that the amino-acid differences they identify have a "significantly enhanced likelihood of causing ... phenotypic effects", their analyses by no means prove that an amino-acid substitution has functional consequences or adaptive value. Such questions can be answered only by investigations of the proteins in question.

So what do we learn from the mammoth genome, except that sequencing of complete genomes from extinct species is indeed possible and that there are differences in their DNA sequences compared with those of living animals? As with many draft genome projects, not that much. But a draft genome is only the beginning of the story. The main feature of genome projects is to provide a resource for further research, as vividly shown by the thousands of times the initial human-genome sequencing papers ${ }^{8,9}$ have been cited.

The next draft nuclear genome of an extinct species likely to become available is that of our closest relative, the Neanderthal, following on from publication of a complete Neanderthal mitochondrial genome sequence ${ }^{10}$. For some time yet, much work in genomics will consist of fully annotating and completing genome sequences, as indeed most published sequences of extant vertebrates, let alone that of the extinct mammoth, remain drafts. But when we look further into the future, the task will be to understand which differences at the sequence level underlie the phenotypic differences between a mammoth and an elephant, or a human and a Neanderthal, for which well-annotated genomes provide the essential basis.

Michael Hofreiter is at the Max Planck Institute for Evolutionary Anthropology, Deutscher Platz 6, D-04103 Leipzig, Germany.

e-mail: hofreite@eva.mpg.de

1. Miller, W. et al. Nature 456, 387-390 (2008).

2. Sanger, F., Nicklen, S. \& Coulson, A. R. Proc. Natl Acad. Sci. USA 74, 5463-5467 (1977).

3. Margulies, M. et al. Nature $437,376-380$ (2005).

4. Smith, D. R. et al. Genome Res. 18, 1638-1642 (2008).

5. Poinar, H. N. et al. Science 311, 392-394 (2006).

6. Noonan, J. P. et al. Science 309, 597-599 (2005).

7. Rohland, N. et al. PLoS Biol. 5, e207 (2007).

8. International Human Genome Sequencing Consortium Nature 409, 860-921 (2001).

9. Venter, J. C. et al. Science 291, 1304-1351 (2001)

10. Green, R. E. et al. Cell 134, 416-426 (2008).

\title{
PALAEOCLIMATE
}

\section{Greenhouse-gas fingerprints}

\author{
Thomas F. Stocker and Adrian Schilt
}

\section{Short episodes of warming and cooling occurred throughout the last glaciation. An innovative modelling study indicates that ocean-circulation changes produced much of the causative variation in greenhouse gases.}

Much of what we know about abrupt climate change and tipping points in the climate system comes from polar ice cores ${ }^{1}$. But these unique data archives provide only a narrow view of the richness of climate dynamics and impacts. Moreover, the origin of the variations in the greenhouse gases associated with the pronounced climate swings during the last ice age, an interval between about 110,000 and 10,000 years ago, remains largely unknown. Hence the significance of computer models in providing a wider perspective.

On page 373 of this issue, Schmittner and Galbraith ${ }^{2}$ present climate-model simulations for an episode of abrupt climate change during the last ice age. Their results show agreement with the palaeoclimatic record ${ }^{3}$, not only in terms of physical climate variables, but also, remarkably, in changes in the greenhouse gases carbon dioxide $\left(\mathrm{CO}_{2}\right)$ and nitrous oxide $\left(\mathrm{N}_{2} \mathrm{O}\right)$. The researchers conclude that the interaction of physical and biogeochemical processes in the ocean is largely responsible for the observed variations.

Schmittner and Galbraith ${ }^{2}$ use a coupled climate model of intermediate complexity suitable for palaeoclimatic studies. The physical part of the simulation features a comprehensive ocean-circulation model coupled to an energy-balance model of the atmosphere. A marine-ecosystem module, which includes two classes of phytoplankton, simulates the distribution of nitrate, phosphate, oxygen, inorganic carbon and alkalinity in the ocean. Although the model accounts for carbon cycling in the ocean, atmosphere and terrestrial vegetation, it deals with the global nitrogen cycle only in a simplified fashion. Production of marine $\mathrm{N}_{2} \mathrm{O}$ is diagnosed from oxygen concentrations, and the stratospheric sink and the soil source of $\mathrm{N}_{2} \mathrm{O}$ are assumed to remain constant.

During the last ice age, the climate system exhibited a series of rapid changes known as Dansgaard-Oeschger events. These involved temperature changes of up to $15^{\circ} \mathrm{C}$ in Greenland within a few decades ${ }^{4}$. There is good 